\title{
Medio de cultivo líquido para el diagnóstico de paratuberculosis bovina. Aplicación y análisis comparativo con el medio de Herrold: resultados preliminares
}

\author{
Liquid culture medium for diagnosis of bovine paratuberculosis. \\ Usefulness and comparative analysis with Herrold medium: preliminary \\ results
}

\author{
Romero Mi,2*, Alvarado Pinedo M², Moyano R33,4, Peralta L², Sosa P², \\ Santangelo M3,4, Travería G ${ }^{2}$
}

1. Comisión de Investigaciones Científicas de la Provincia de Buenos Aires (CICPBA). 2. Centro de Diagnóstico e Investigaciones Veterinarias, FCV, UNLP (CEDIVE, Chascomús). 3. Consejo Nacional de Investigaciones Científicas y Técnicas (CONICET). 4. Instituto Nacional de Tecnología Agropecuaria (INTA), Instituto de Biotecnología, Castelar. Argentina

* Correo electrónico de la autora de contacto: mromero@fcv.unlp.edu.ar

\begin{abstract}
Resumen
La paratuberculosis es una enfermedad crónica producida por un bacilo ácido-alcohol resistente (BAAR), el Mycobacterium avium subsp. paratuberculosis (Map). No existe tratamiento ni vacuna aprobada por SENASA para esta enfermedad, por lo que es imprescindible el diagnóstico de los animales positivos para controlar la enfermedad antes de que infecten a otros. Se probó la implementación de un medio de cultivo líquido para el diagnóstico de paratuberculosis en bovinos, modificado a partir del medio Middlebrook $\mathrm{M} 7 \mathrm{H} 9$ caldo. Para probar la efectividad del medio se realizó la siembra pareada de muestras de materia fecal y tejidos animales en el medio de cultivo sólido de Herrold con micobactina (HEYM) y en el medio de cultivo líquido modificado a partir del $\mathrm{M} 7 \mathrm{H} 9$ caldo. Además, se lograron comparar el tiempo de detección de colonias en el medio sólido con el de detección de BAAR formando grupos en el medio de cultivo líquido en estudio. Se corroboró que hay diferencias significativas entre el medio de cultivo líquido y el medio HEYM, tanto en el tiempo de detección como en la cantidad de muestras positivas detectadas, observando BAAR en el medio líquido o colonias bacterianas en el medio HEYM. Sobre la base de estos resultados se concluye que el medio líquido probado posee una mayor sensibilidad analítica y un tiempo de detección del crecimiento menor con respecto a los del medio HEYM.
\end{abstract}

\section{Palabras clave}

Micobacterias, medio de cultivo líquido, Ziehl-Neelsen, micobactina

\begin{abstract}
Paratuberculosis is a chronical disease caused by acid-fast bacilli (AFB) Mycobacterium avium subsp. paratuberculosis (Map). There is neither an effective treatment nor any vaccine approved by the sanitary authority (SENASA) in Argentina. Diagnosis and segregation of positive animals are essential for the control of the disease. For the diagnosis of cattle paratuberculosis, a modified Middlebrook $\mathrm{M} 7 \mathrm{H} 9$ broth medium was developed. To test the effectiveness of the medium, fecal and tissue samples were cultured in Herrold with mycobactin and liquid culture media, and time to growth was compared by direct observation of colonies and microscopic grouped AFB observation, respectively. Significant differences were observed between liquid tested and Herrold media, both in time to grow and in the number of positive animals detected. Based on these results, it is concluded that liquid medium offers greater analytical sensitivity and faster detection than Herrold medium.
\end{abstract}

\section{Key words}

Mycobacterium, liquid culture medium, Ziehl-Neelsen, mycobactin

Fecha de recepción: 01/12/2017

Fecha de aprobación: 16/03/2018
ANALECTA VET 2018; 38(1): 50-55

Impresa ISSN 03655 14-8 Electrónica ISSN 1514-2590 


\section{Introducción}

La paratuberculosis, causada por el Mycobacterium avium subsp. paratuberculosis (Map), se caracteriza por provocar una ileocolitis granulomatosa, crónica y debilitante en los animales que la padecen. Es una enfermedad que tiene un gran impacto productivo y económico en el ámbito mundial y en nuestro país, principalmente en producciones lecheras (Morsella \& Paolicci, 2008; Soto et al., 2002). La particularidad de esta enfermedad es que no cuenta con tratamientos eficaces ni métodos profilácticos aprobados por el SENASA hasta el momento, debido a que podrían interferir con el diagnóstico de la tuberculosis. Por lo tanto, su control se basa en el diagnóstico de los animales infectados y su segregación. El diagnóstico puede realizarse determinando la magnitud de la respuesta inmune celular o humoral y, de forma directa, mediante estudios microbiológicos. Entre las posibilidades diagnósticas, el cultivo bacteriológico es considerado la técnica de oro, aun con las dificultades que presentan las micobacterias de crecimiento lento. El medio de cultivo utilizado comúnmente es el de Herrold con yema de huevo y el agregado de micobactina (HEYM), ya que cumple con las exigencias del Map para su crecimiento in vitro (Merkal \& Curran, 1974). Este medio de cultivo presenta una especificidad del $99 \%$ y una sensibilidad cercana al $50 \%$. La decontaminación previa con cloruro de hexadecil-piridinio (HPC) elimina la mayoría de las micobacterias no patógenas, con escasos efectos dañinos sobre el Map (Collins et al., 1996; Whipple et al., 1991). Otro inconveniente que se suma a la baja sensibilidad del medio HEYM es que la aparición de colonias requiere entre dos y cuatro meses de incubación, y solo luego de seis meses es posible asegurar un resultado negativo (Stabel et al., 1997; Whipple et al., 1991). Los tiempos de incubación prolongados limitan la implementación del cultivo bacteriológico, incluso cuando se lo usa con fines de investigación. Debido a todas las dificultades que presenta el cultivo micobacteriano, en un intento por abreviar el lapso hasta la constatación del crecimiento bacteriano y por mejorar la sensibilidad del método, se han probado diversos medios de cultivo, habiéndose obtenido mejores resultados con el uso de medios de cultivo líquidos (Pozzato et al., 2011; Stabel et al., 1997; Whittington, 2009). Así se han logrado incluso tipificar las cepas aisladas a partir de ellos (Motiwala et al., 2005). Sin embargo, estos medios comerciales no están disponibles en el país y su adquisición resulta actualmente dificultosa y de alto costo.

En este trabajo se probó la preparación e implementación de un medio de cultivo líquido modificado a partir del medio líquido $\mathrm{M} 7 \mathrm{H} 9 \mathrm{C}$ (Whittington et al., 2013). Se comparó su eficacia, con respecto al tiempo hasta la constatación del crecimiento bacteriano y la sensibilidad analítica, en comparación con el medio sólido HEYM.

\section{Descripción metodológica}

\section{Medios de cultivo}

-Medio de Herrold con yema de huevo y micobactina (HEYM):

Los componentes de la preparación se detallan en la tabla 1. Se fraccionó en botellas de cultivo celular de $50 \mathrm{ml}$ colocando $10 \mathrm{ml}$ de medio en cada una de ellas.

\section{-Medio de cultivo líquido:}

Se preparó según los componentes detallados en la tabla 1. Se basó en el medio de cultivo descripto por Whittington et al. (2013) al que se le agregó agar al 0,185 \% para que se mantuviera el estado líquido del medio, pero colaborara con la homogeneidad de la yema de huevo en la columna. Además, se agregó ácido nalidíxico y nistatina para evitar la contaminación. Este medio se fraccionó en tubos de vidrio de 30 $\mathrm{ml}$ conteniendo $10 \mathrm{ml}$ de medio cada uno.

Se procesaron 47 muestras provenientes de bovinos con y sin signos de la enfermedad (Tabla 2). Las muestras de materia fecal se recolectaron directamente del recto de los animales. También se procesaron muestras de tres órganos (íleon, yeyuno y linfonódulo) obtenidas durante la necropsia de animales con signos compatibles con la enfermedad. Las necropsias fueron realizadas por nuestro grupo de trabajo en el establecimiento.

\section{Procesamiento de las muestras}

Se realizó la decontaminación de acuerdo con el método de doble incubación (Stabel, 1997) colocando $2 \mathrm{~g}$ de muestra (materia fecal o tejido, según el caso) en $30 \mathrm{ml}$ de agua destilada estéril. Estas muestras luego se agitaron y dejaron reposar durante 30 minutos. Posteriormente se colocaron $5 \mathrm{ml}$ del sobrenadante en $25 \mathrm{ml}$ de solución de HPC y esta solución se mantuvo en estufa a $37^{\circ} \mathrm{C}$ durante $24 \mathrm{~h}$. Se centrifugó a temperatura ambiente durante 30 minutos a $1000 \mathrm{rpm}$, se descartó el sobrenadante, se agregó $1 \mathrm{ml}$ de mezcla antibiótica y se conservó $24 \mathrm{~h}$ a $37^{\circ} \mathrm{C}$. Las muestras así procesadas se sembraron, en forma pareada, colocando $800 \mu \mathrm{l}$ en las botellas con HEYM y $100 \mu \mathrm{l}$ (Whittington et al., 2013) en los tubos con el medio de cultivo líquido en estudio. Ambos fueron incubados en estufa a $37^{\circ} \mathrm{C}$.

Seguimiento de las muestras en ambos medios de cultivo y criterios de positividad

Se observaron ambos medios de cultivo semanalmente. En el HEYM se consideraron positivas aquellas muestras que evidenciaron al 
menos una colonia blanquecina, lisa, brillante y visible macroscópicamente (Fig. 1). La observación del medio de cultivo líquido se realizó microscópicamente, utilizando la coloración de Ziehl-Neelsen para poner de manifiesto bacilos ácido-alcohol resistentes (BAAR). Se consideraron positivas aquellas muestras que mostraron BAAR agrupados y morfológicamente compatibles con Map (Fig. 2). Las coloraciones se realizaron semanalmente y se inspeccionaron 50 campos de observación, como mínimo, cada semana.

\begin{tabular}{|c|c|c|c|}
\hline Ingredientes & A & B & c \\
\hline base medio M7H9C & $3,1 \mathrm{~g}$ & $3,1 \mathrm{~g}$ & \\
\hline albúmina bovina & $1,34 \mathrm{~g}$ & $1,6 \mathrm{~g}$ & \\
\hline dextrosa & $0,52 \mathrm{~g}$ & $0,64 \mathrm{~g}$ & \\
\hline catalasa & $0,8 \mathrm{mg}$ & $0,96 \mathrm{mg}$ & \\
\hline Bacto Casitone ${ }^{\circledast}$ & $666 \mathrm{mg}$ & $680 \mathrm{mg}$ & \\
\hline cloruro de sodio & $0,22 \mathrm{~g}$ & $0,22 \mathrm{~g}$ & $15,3 \mathrm{~g}$ \\
\hline yema de huevo & $167 \mathrm{ml}$ & $170 \mathrm{ml}$ & $120 \mathrm{ml}$ \\
\hline Micobactina J๑ & $0,8 \mathrm{mg}$ & $1 \mathrm{mg}$ & $2 \mathrm{mg}$ \\
\hline Panta plus ${ }^{\ominus}$ & $30 \mathrm{ml}$ & $30 \mathrm{ml}$ & \\
\hline ácido nalidíxico & & $200 \mathrm{mg}$ & $100 \mathrm{mg}$ \\
\hline nistatina & & $800 \mathrm{mg}$ & $400 \mathrm{mg}$ \\
\hline vancomicina & & & $100 \mathrm{mg}$ \\
\hline agar & & $1,8 \mathrm{~g}$ & $15,3 \mathrm{~g}$ \\
\hline peptona de carne & & & $9 \mathrm{~g}$ \\
\hline piruvato de sodio & & & $4,1 \mathrm{~g}$ \\
\hline extracto de carne & & & $2,7 \mathrm{~g}$ \\
\hline glicerol & & & $34 \mathrm{ml}$ \\
\hline verde de malaquita al $2 \%$ & & & $5,2 \mathrm{ml}$ \\
\hline agua tridestilada & $800 \mathrm{ml}$ & $770 \mathrm{ml}$ & $840,9 \mathrm{ml}$ \\
\hline
\end{tabular}

Tabla 1. Composición (por litro) de cada medio. A. Medio descripto por Whittington et al. (2013). B. Medio líquido experimental del trabajo. C. Medio sólido HEYM.

\section{Confirmación de resultados}

Para el caso del medio de cultivo líquido se procedió a confirmar la presencia de Map en las muestras positivas mediante la técnica de análisis molecular de reacción en cadena de la polimerasa (PCR). El segmento de inserción que se buscó amplificar fue IS90o (Collins et al., 1993). Las muestras positivas en el HEYM fueron coloreadas con la técnica de Ziehl-Neelsen y procesadas mediante PCR IS9oo.

\section{Recuperación y extracción de ADN para PCR IS9OO}

Debido a la presencia de inhibidores del crecimiento en el huevo, componente del medio de cultivo líquido en estudio, se debió realizar un repique en medio Löwenstein-Jensen (con micobactina), donde se observó el desarrollo de colonias entre los 5 y los 10 días. A partir de este medio se tomó una colonia y se diluyó en $200 \mu \mathrm{l}$ de agua ultra pura para PCR, sometiéndola a 3 ciclos de hervido/congelación (shock térmico) para la extracción de ADN. Las muestras fueron procesadas mediante PCR IS90o siguiendo el protocolo de Collins et al. (1993). Los cebadores utilizados fueron: hacia adelante (F) GATCGGAACGTCGGCTGGTCAGG y reverso (R) GATCGCCTTGCTCATCGCTGCCG, utilizando DNA Polymerase
BIOLINEß (Londres, Reino Unido) y termociclador Evo MPI M-02@ (La Plata, Argentina). Se obtuvo un producto de $218 \mathrm{pb}$.

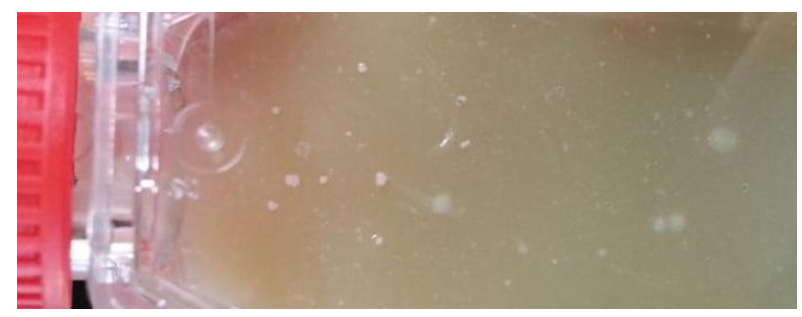

Figura 1. Colonias típicas de Map, blanquecinas, lisas, brillantes. Medio HEYM, 70 días posincubación (muestra $\mathrm{N}^{\circ}$ 43).

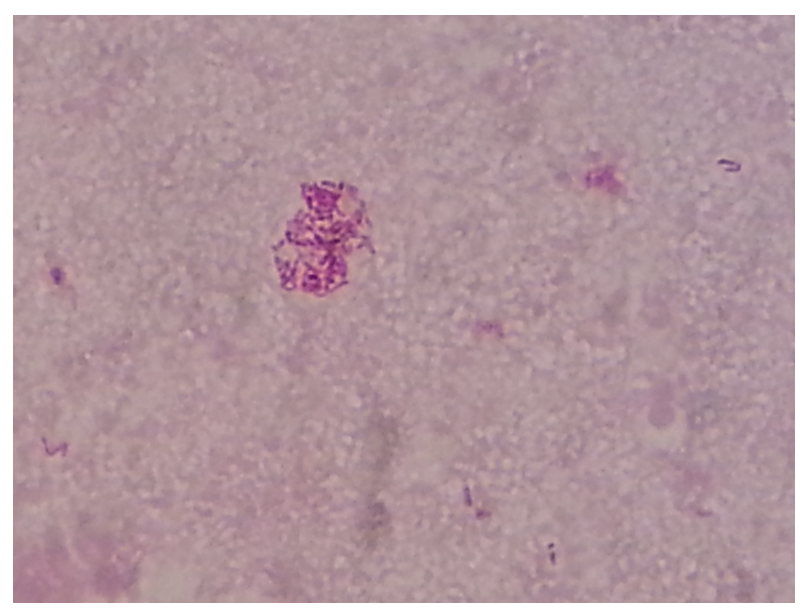

Figura 2. Bacilos ácido-alcohol resistentes compatibles con Map, medio líquido, 17 días posincubación. Coloración de ZiehlNeelsen (muestra $\mathrm{N}^{\circ} 43$ ).

\section{Análisis de datos}

Se analizó la variable resultados en el medio de cultivo líquido en estudio respecto del HEYM mediante la prueba de chi cuadrado con corrección de Yates, y se obtuvo un valor de $\mathrm{p}=$ 0,002 (intervalo de confianza -IC- $95 \%$ ). También se utilizó la prueba de T de Student para analizar las diferencias en cuanto al tiempo de detección de crecimiento en ambos medios de cultivo, mediante la cual se encontraron diferencias con valor de $\mathrm{p}=$ 0,00000000005 (IC 95 \%). Tanto el chi cuadrado como la prueba de $\mathrm{T}$ fueron realizadas mediante programa Excel, Windows 8.1. Para las mismas muestras se puso en evidencia una mayor cantidad de casos positivos en el medio líquido que en el medio sólido. Las muestras positivas en el medio líquido fueron $34 \mathrm{y}$, en el medio HEYM, 22 (diferencias estadísticas con un valor de $\mathrm{p}=0,002$, IC $95 \%)$. Mediante la técnica de PCR IS90o se confirmaron 25 de las 34 muestras positivas en el medio líquido, 8 de las cuales habían resultado negativas en el medio HEYM. 


\begin{tabular}{|c|c|c|c|c|c|c|c|c|c|c|}
\hline \multirow[b]{2}{*}{$\mathbf{N}^{\circ}$} & \multirow[b]{2}{*}{$\begin{array}{l}\text { Tipo de } \\
\text { muestra }\end{array}$} & \multirow[b]{2}{*}{ Año* } & \multirow[b]{2}{*}{ Categoría } & \multirow[b]{2}{*}{ Raza } & \multirow[b]{2}{*}{ Signos clínicos } & \multirow[b]{2}{*}{ Zona de procedencia } & \multicolumn{2}{|c|}{ Medio líquido } & \multicolumn{2}{|c|}{ Medio de Herrold } \\
\hline & & & & & & & Rdo & $\begin{array}{l}\text { Tiempo } \\
\text { (días) }\end{array}$ & Rdo & $\begin{array}{l}\text { Tiempo } \\
\text { (días) }\end{array}$ \\
\hline 1 & MF & 2016 & Vaca & AA & $\mathrm{Si}$ & Magdalena, Bavio & POSITIVO & 8 & POSITIVO & 60 \\
\hline 7 & MF & 2016 & Vaca & AA & $\mathrm{Si}$ & Vieytes & POSITIVO & 19 & POSITIVO & 111 \\
\hline 13 & MF & 2016 & Vaca & AA & $\mathrm{Si}$ & Azul & POSITIVO & 12 & POSITIVO & 50 \\
\hline 14 & MF & 2016 & Vaca & AA & $\mathrm{Si}$ & Azul & POSITIVO & 19 & POSITIVO & 70 \\
\hline 15 & MF & 2016 & Vaca & AA & $\mathrm{Si}$ & Azul & POSITIVO & 8 & POSITIVO & 67 \\
\hline 17 & MF & 2016 & Vaca & AA & $\mathrm{Si}$ & Magdalena, Bavio & POSITIVO & 4 & POSITIVO & 70 \\
\hline 19 & MF & 2015 & Vaca & AA & $\mathrm{Si}$ & Vieytes & POSITIVO & 17 & POSITIVO & 58 \\
\hline 32 & MF & 2016 & Vaca & HA & $\mathrm{Si}$ & Tandil & POSITIVO & 5 & POSITIVO & 40 \\
\hline 33 & MF & 2016 & Vaca & AA & $\mathrm{Si}$ & Gral. Belgrano & POSITIVO & 5 & POSITIVO & 32 \\
\hline 34 & MF & 2016 & Vaca & AA & $\mathrm{Si}$ & Chascomús & POSITIVO & 6 & POSITIVO & 58 \\
\hline 35 & MF & 2016 & Vaca & AA & $\mathrm{Si}$ & Vieytes & POSITIVO & 19 & POSITIVO & 49 \\
\hline 36 & MF & 2016 & Vaquillona & AA & $\mathrm{Si}$ & La Plata & POSITIVO & 12 & POSITIVO & 49 \\
\hline 37 & MF & 2016 & Vaca & AA & $\mathrm{Si}$ & Brandsen & POSITIVO & 7 & POSITIVO & 106 \\
\hline 38 & MF & 2016 & Vaca & HA & $\mathrm{Si}$ & Tandil & POSITIVO & 14 & POSITIVO & 72 \\
\hline 39 & MF & 2016 & Vaca & HA & $\mathrm{Si}$ & Tandil & POSITIVO & 13 & POSITIVO & 58 \\
\hline 40 & MF & 2015 & Vaca & AA & $\mathrm{Si}$ & Chascomús & POSITIVO & 8 & POSITIVO & 56 \\
\hline 41 & MF & 2015 & Vaca & AA & $\mathrm{Si}$ & Chascomús & POSITIVO & 8 & POSITIVO & 58 \\
\hline 43 & Yeyuno & 2015 & Vaca & AA & $\mathrm{Si}$ & Vieytes & POSITIVO & 17 & POSITIVO & 52 \\
\hline 44 & Íleon & 2015 & Vaca & AA & $\mathrm{Si}$ & Vieytes & POSITIVO & 17 & POSITIVO & 122 \\
\hline 45 & $\mathrm{MF}$ & 2016 & Vaca & AA & $\mathrm{Si}$ & Azul & POSITIVO & 12 & POSITIVO & 87 \\
\hline 46 & MF & 2016 & Vaca & AA & $\mathrm{Si}$ & Azul & POSITIVO & 8 & POSITIVO & 50 \\
\hline 47 & MF & 2016 & Vaca & AA & $\mathrm{Si}$ & Gral. Belgrano & POSITIVO & 5 & POSITIVO & 60 \\
\hline 2 & MF & 2016 & Vaquillona & AA & No & Gral. Belgrano & POSITIVO & 35 & NEGATIVO & \\
\hline 3 & $\mathrm{MF}$ & 2016 & Vaquillona & AA & No & Gral. Belgrano & POSITIVO & 49 & NEGATIVO & \\
\hline 4 & MF & 2016 & Vaquillona & AA & No & Gral. Belgrano & POSITIVO & 5 & NEGATIVO & \\
\hline 5 & $\mathrm{MF}$ & 2016 & Vaca & AA & $\mathrm{Si}$ & Gral. Belgrano & POSITIVO & 5 & NEGATIVO & \\
\hline 6 & $\mathrm{MF}$ & 2016 & Vaca & AA & $\mathrm{Si}$ & Gral. Belgrano & POSITIVO & 5 & NEGATIVO & \\
\hline 8 & $\mathrm{MF}$ & 2016 & Vaca & AA & No & Vieytes & POSITIVO & 14 & NEGATIVO & \\
\hline 9 & MF & 2015 & Vaca & AA & $\mathrm{Si}$ & Vieytes & POSITIVO & 17 & NEGATIVO & \\
\hline 10 & Linfonodo & 2015 & Vaca & AA & $\mathrm{Si}$ & Vieytes & POSITIVO & 50 & NEGATIVO & \\
\hline 11 & $\mathrm{MF}$ & 2016 & Vaca & AA & $\mathrm{Si}$ & Chascomús & POSITIVO & 20 & NEGATIVO & \\
\hline 12 & $\mathrm{MF}$ & 2016 & Vaca & AA & $\mathrm{Si}$ & Chascomús & POSITIVO & 13 & NEGATIVO & \\
\hline 16 & $\mathrm{MF}$ & 2016 & Vaca & AA & $\mathrm{Si}$ & Azul & POSITIVO & 6 & NEGATIVO & \\
\hline 18 & MF & 2016 & Vaca & AA & $\mathrm{Si}$ & La Plata & POSITIVO & 12 & NEGATIVO & \\
\hline 42 & MF & 2015 & Vaca & AA & $\mathrm{Si}$ & Chascomús & NEGATIVO & & POSITIVO & 69 \\
\hline 20 & MF & 2015 & Vaca & AA & $\mathrm{Si}$ & Chascomús & NEGATIVO & & NEGATIVO & \\
\hline 21 & MF & 2016 & Vaca & AA & $\mathrm{Si}$ & Vieytes & NEGATIVO & & NEGATIVO & \\
\hline 22 & MF & 2016 & Vaca & AA & $\mathrm{Si}$ & Luján & NEGATIVO & & NEGATIVO & \\
\hline 23 & MF & 2016 & Vaca & AA & $\mathrm{Si}$ & Gral. Belgrano & NEGATIVO & & NEGATIVO & \\
\hline 24 & MF & 2016 & Vaca & AA & $\mathrm{Si}$ & Gral. Belgrano & NEGATIVO & & NEGATIVO & \\
\hline 25 & MF & 2016 & Vaca & HA & $\mathrm{Si}$ & Tandil & NEGATIVO & & NEGATIVO & \\
\hline 26 & MF & 2016 & Vaca & HA & $\mathrm{Si}$ & Tandil & NEGATIVO & & NEGATIVO & \\
\hline 27 & MF & 2016 & Vaca & HA & $\mathrm{Si}$ & Tandil & NEGATIVO & & NEGATIVO & \\
\hline 28 & MF & 2016 & Vaca & HA & $\mathrm{Si}$ & Tandil & NEGATIVO & & NEGATIVO & \\
\hline 29 & MF & 2016 & Vaca & HA & $\mathrm{Si}$ & Tandil & NEGATIVO & & NEGATIVO & \\
\hline 30 & MF & 2016 & Vaca & AA & $\mathrm{Si}$ & Chascomús & NEGATIVO & & NEGATIVO & \\
\hline 31 & MF & 2016 & Vaca & AA & $\mathrm{Si}$ & Chascomús & NEGATIVO & & NEGATIVO & \\
\hline
\end{tabular}

Tabla 2. Origen y características de las muestras, resultados y tiempos de detección en medio de cultivo líquido y medio de cultivo de Herrold. MF: Materia fecal. Rdo: Resultado. AA: Aberdeen Angus. HA: Holando Argentino. *Año en el que se tomó y procesó la muestra. 
Las 9 muestras restantes se contaminaron, dieron resultado negativo y no pudieron ser recuperadas para un nuevo procesamiento mediante PCR. Sin embargo, todas ellas mostraron características morfológicas típicas de Map con la coloración de Ziehl-Neelsen, incluyendo el agrupamiento clásico de BAAR. Cinco de ellas, además, resultaron positivas en el medio HEYM, con el desarrollo de colonias de morfología clásica. A su vez, las muestras positivas en el medio HEYM fueron corroboradas en su totalidad mediante la coloración de Ziehl-Neelsen y con la amplificación del segmento IS900, aunque en dos de los casos (muestras 37 y 44) la banda resultante en el gel de corrida electroforética fue débil respecto de las demás y podrían considerarse negativas.

En cuanto al tiempo de detección de crecimiento, el promedio para el medio HEYM fue de 65 días, mientras que para el medio de cultivo líquido fue de 11 días (Tabla 2). Estos datos arrojaron un grado de significancia de $\mathrm{p}=0,00000000005$ (IC 95 \%) mediante la prueba de T, mostrando que existen diferencias significativas también para esta variable.

\section{Discusión y conclusiones}

Con el uso del medio líquido modificado a partir del medio $\mathrm{M} 7 \mathrm{H} 9 \mathrm{C}$ (Whittington et al., 2013) fue posible mejorar notablemente, tanto la sensibilidad analítica del cultivo como así también el tiempo de incubación, marcadamente menor que el requerido con el medio HEYM, utilizado de rutina. Estos resultados son coincidentes con los de trabajos previos que demuestran la mayor sensibilidad de los medios de cultivo líquidos y que también describen un desarrollo en menor tiempo (Pozzato et al., 2011; Stabel et al., 1997; Shin et al., 2007; Whittington, 2009, 2013). La observación de crecimiento diagnosticable de Map en el cultivo en un promedio de 11 días fue algo destacable, teniendo en consideración que se trata de una micobacteria de crecimiento lento. Sin embargo, Shin et al. (2007) describen los tiempos de detección de crecimiento y consignan que, en los sistemas BACTEC 460 y MGIT, muestras con 105 $\mathrm{UFC} / \mathrm{ml}$ resultan positivas 7 u 8 días posincubación. Teniendo en cuenta este dato y considerando que la mayor parte de las muestras de este trabajo provenían de animales con signos clínicos (y que, por lo tanto, se encuentran eliminando micobacterias en grandes cantidades), es posible afirmar que los resultados del trabajo aquí presentado se encuentran dentro de lo esperable.

En cuanto a la mayor sensibilidad analítica que mostró el medio de cultivo líquido respecto del sólido, cabe aclarar que la utilización de la presencia de BAAR como criterio de positividad posibilita la inclusión errónea de otros BAAR (diferentes de Map), alterando la especificidad del cultivo. Para determinar la presencia de Map se utilizó la técnica de PCR IS900, que nos permitió corroborar los resultados de gran parte de las muestras y confirmar alrededor del $66 \%$ de las muestras que resultaron positivas en el medio líquido, pero no en el HEYM, utilizado de rutina como técnica de oro. Si bien es necesario aumentar la cantidad de muestras procesadas para robustecer el soporte estadístico, los resultados obtenidos son prometedores, y permitirían dar respuesta a la necesidad de mejorar las características del cultivo micobacteriano para poder brindar un mejor servicio de diagnóstico. Una mayor cantidad de muestras procesadas eventualmente permitirán decidir si es factible proponer al cultivo en este medio líquido como una alternativa superadora del realizado en medio HEYM, con respecto al tiempo de incubación y a la sensibilidad, sin disminuir su especificidad.

\section{Agradecimientos}

Este trabajo fue financiado mediante beca de la Comisión de Investigaciones Científicas de la Provincia de Buenos Aires.

\section{Conflicto de intereses} declarar.

No existe conflicto de intereses que

\section{Bibliografía}

Andrews AH. 2004. Bovine medicine: diseases and husbandry of cattle. $2^{\circ}$ Ed. Reimp. Reino Unido, Blackwell Publishing Company.

Collins DM, Stephens DM, de Lisle GW. 1993. Comparison of polymerase chain reaction tests and faecal culture for detecting Mycobacterium paratuberculosis in bovine faeces. Veterinary Microbiology. 36(3-4):288-99.

Collins, MT. 1996. Diagnosis of paratuberculosis. The Veterinary Clinics of North America: Food Animal Practice. 12(2):357-71.

Merkal RS, Curran BJ. 1974. Growth and metabolic characteristics of Mycobacterium paratuberculosis. Applied Microbiology. 28:2769.

Morsella C, Paolicchi F. 2008. Aplicación de métodos de diagnóstico y control de paratuberculosis en Argentina. XXI Congreso Panamericano de Ciencias Veterinarias. Guadalajara, México, pp. 37-43.

Motiwala AS, Strother M, Theus NE, Stich RW, Byrum B, Shulaw WP, Kapur V, Sreevatsan S. 2005. Rapid detection and typing of strains of Mycobacterium avium subsp. paratuberculosis 
from broth cultures. Journal of Clinical Microbiology. 43(5):2111-7.

doi: 10.1128/JCM.43.5.2111-2117.2005

Pozzato N, Gwozdz J, Gastaldelli M, Capello K, Ben CD, Stefani E. 2011. Evaluation of a rapid and inexpensive liquid culture system for the detection of Mycobacterium avium subsp. paratuberculosis in bovine faeces. Journal of Microbiological Methods. 84(3):413-7.

doi: 10.1016/j.mimet.2011.01.019

Shin SJ, Han JH, Manning EJ, Collins MT. 2007. Rapid and reliable method for quantification of Mycobacterium paratuberculosis by use of the BACTEC MGIT 960 system. Journal of Clinical Microbiology. 45(6):1941-8.

doi:10.1128/JCM.02616-06

Soto JP, Kruze J, Leiva S. 2002. Aislamiento de Mycobacterium avium subsp. paratuberculosis de fecas en rebaños lecheros infectados mediante el método de Cornell modificado. Archivos de Medicina Veterinaria. 34(2):275-82.

doi: 10.4067/So301-732X2002000200013

Stabel JR. 1997. An improved method for cultivation of Mycobacterium paratuberculosis from bovine fecal samples and comparison to three other methods. Journal of Veterinary Diagnostic Investigation. 9(4) 375-80.

Whipple DL, Callihan DR, Jarnagin JL. 1991. Cultivation of Mycobacterium paratuberculosis from bovine fecal specimens and a suggested standardized procedure. Journal of Veterinary Diagnostic Investigation. 3(4):368-73.

doi: 10.1177/104063879100300424

Whittington RJ. 2009. Factors affecting isolation and identification of Mycobacterium avium subsp. paratuberculosis from fecal and tissue samples in a liquid culture system. Journal of Clinical Microbiology. 47(3):614-22.

doi: 10.1128/JCM.01986-08

Whittington RJ, Whittington AM, Waldron A, Begg DJ, De Silva K, Purdie AC, Plain KM. 2013. Development and validation of a liquid medium (M7H9C) for routine culture of Mycobacterium avium subsp. paratuberculosis to replace modified Bactec 12B medium. Journal of Clinical Microbiology. 51(12):3993-4000.

doi: 10.1128/JCM.01373-13 